\title{
Precision mass measurements of very short-lived, neutron-rich Na isotopes using a radio-frequency spectrometer
}

\author{
D. Lunney, ${ }^{*}$ G. Audi, H. Doubre, S. Henry, C. Monsanglant, M. de Saint Simon, C. Thibault, and C. Toader ${ }^{\dagger}$ \\ Centre de Spectrométrie Nucléaire et de Spectrométrie de Masse (CSNSM), IN2P3/CNRS-Université Paris Sud, Bâtiment 108, \\ F-91405 Orsay Campus, France \\ C. Borcea ${ }^{\ddagger}$ \\ Institute for Atomic Physics, Bucharest, Romania \\ G. Bollen ${ }^{\S}$ and the ISOLDE Collaboration \\ CERN, CH-1211 Geneva, Switzerland
}

(Received 24 April 2001; published 12 October 2001)

\begin{abstract}
Mass measurements of high precision have been performed on sodium isotopes out to ${ }^{30} \mathrm{Na}$ using a new technique of radio-frequency excitation of ion trajectories in a homogeneous magnetic field. This method, especially suited to very short-lived nuclides, has allowed us to significantly reduce the uncertainty in mass of the most exotic Na isotopes: a relative error of $5 \times 10^{-7}$ was achieved for ${ }^{28} \mathrm{Na}$ having a half-life of only 30.5 $\mathrm{ms}$ and $9 \times 10^{-7}$ for the weakly produced ${ }^{30} \mathrm{Na}$. Verifying and minimizing binding energy uncertainties in this region of the nuclear chart is important for clarification of a long-standing problem concerning the strength of the $N=20$ magic shell closure. These results are the fruit of the commissioning of the new experimental program MISTRAL.
\end{abstract}

DOI: 10.1103/PhysRevC.64.054311

PACS number(s): 21.10.Dr, 27.30.+t, 29.30.Aj

\section{INTRODUCTION}

The atomic mass is a global property that reflects the net result of all interactions at work in the atom. Information concerning nuclear structure can be derived from examination of the binding energies of various nuclear configurations over the so-called mass surface [1].

One way the mass surface can be defined is by the twoneutron separation energy $S_{2 n}$, the difference in binding energy (BE) between two isotopes $\mathrm{BE}(Z, N)$ and $\mathrm{BE}(Z, N$ -2 ), versus $N$. The $S_{2 n}$ surface has a general trend of linearly decreasing separation energy with neutron number; the more neutrons in the nucleus, the less they are bound. Deviations from this behavior point to manifestations of nuclear structure, one of the most striking examples of which is shell structure.

Of questions pertinent to nuclear structure raised by masses, shell effects are perhaps the most fundamental. Magic nucleon numbers offer pillars of nuclear stability but it seems that these pillars may be eroded in regions where the ratio of neutrons to protons becomes excessive.

The so-called "island of inversion" was first discovered by Thibault et al. in 1975 [2] when the comportment of $S_{2 n}$ values in sodium showed an abnormal upturn at $N=19$ and above, where a normally stabilizing shell closure would

\footnotetext{
*Email address: lunney@csnsm.in2p3.fr

${ }^{\dagger}$ Also at IAP, Bucharest, Romania.

${ }^{*}$ Present address: CERN, CH-1211, Geneva, Switzerland.

${ }^{\S}$ Also at GSI, D-64291 Darmstadt, Germany. Present address: NSCL, Michigan State University, East Lansing, MI.
}

cause a downturn at $N=21$. This phenomenon was attributed to nuclear deformation effects and has since been the subject of intense study. Not only have further mass measurements been made to map the shores of this curious island [3-7], but other types of complementary measurements have been made to learn more about its topography. Early efforts using laser spectroscopy of $\mathrm{Na}$ isotopes [8] revealed an increase in mean-square-charge radius at $N=18$, consistent with the onset of deformation. $\beta$-spectroscopy studies [9-11] revealed more details of the nuclear level structure, notably a lowlying $2^{+}$excited state in the ${ }^{32} \mathrm{Mg}$ daughter, confirmed by a complementary Coulomb dissociation technique that also provided a quadrupole deformation parameter via the $B(E 2)$ transition probability value [12]. More recent measurements of the quadrupole moments of ${ }^{26-31} \mathrm{Na}$ via $\beta$-NMR and optical polarization have confirmed that strong deformation is indeed present $[13,14]$.

Consequently, considerable theoretical effort has been concentrated on this phenomenon including calculations using Hartree-Fock $[15,16]$, the shell model [17-21], and the relativistic mean field $[22,23]$. Early shell model calculations performed by Chung and Wildenthal [17] failed to reproduce the deformation thought responsible for the inversion phenomenon due to the restricted $s d$ space used whereas later calculations using a more extended basis did succeed $[18,19]$. Interestingly enough, the original mean-field computation of Campi et al. [15] that reproduced deformation of ${ }^{30} \mathrm{Na}$ did so by restoring rotational invariance, something not done in a more recent mean-field calculation of ${ }^{32} \mathrm{Mg}$ [16] which is consequently found to be spherical.

Mass measurements first brought this problem into the arena for study and naturally, masses continue to provide not only further data for nuclear structure but also a very strin- 
gent test for the various theoretical approaches. Modern experimental techniques using intermediate energy exotic beams created either by projectile fragmentation or by the ISOL technique [24] are being employed to glean more experimental data in this and other regions (for example the $N=28$ shell closure $[25,26])$. In the mean time, great progress has also been made in the field of mass spectrometry (see, e.g., Ref. [27]). The MISTRAL experiment (Mass measurements at ISOLDE using a transmission and radiofrequency spectrometer on-line), described in this paper, is one such example. MISTRAL has enabled us to return to the origin of this interesting physics problem by examining, with unprecedented accuracy, the neutron separation energies of the exotic nuclides constituting the island of inversion.

Masses can be determined a variety of ways, notably in the form of differences resulting from the $Q$ value of a radioactive decay [29] or nuclear reaction [30]. While decay measurements can be quite accurate, one must be careful to have complete knowledge of the level scheme in order to correctly determine the ground state and feedings and also to link the resulting mass difference value to a known mass value, sometimes very far away.

Methods that are complementary-and not prone to cumulative error-are based on mass spectrometry via time-offlight or cyclotron frequency measurements. Presently there are several such experimental programs dedicated to the measurement of masses of radioactive ions by mass spectrometry (for reviews, see, Refs. [24,27,28]).

Relatively new on the scene, MISTRAL at ISOLDE uses the radio-frequency excitation of the kinetic energy of an ion beam in a homogeneous magnetic field in order to determine the ion cyclotron frequency and hence, the mass. MISTRAL is capable of high precision (about $0.5 \mathrm{ppm}$ ) and at the same time (given sufficient production) can measure very short lived nuclides since the measurement is made by recording the transmission of the ion beam at its full transport energy $(60 \mathrm{keV})$. The half-life limitation corresponds, not to the short flight time through the apparatus (about $50 \mu \mathrm{s}$ ) but rather the diffusion time of the radioactive species from the ISOLDE thick-target matrix (some ms for the fastest elements).

Thus MISTRAL brings a unique combination of fast measurement time and high precision. This compares to the timeof-flight technique of SPEG and TOFI [4-7,26] (high sensitivity and fast measurement time), the Penning trap spectrometer ISOLTRAP [31] (high sensitivity and high precision), and the Experimental Storage Ring at GSI [32,33] (high sensitivity and now, fast measurement time due to a new isochronous mode of ring operation [34]).

This paper will describe the first results of MISTRAL, masses of the isotopes ${ }^{23-30} \mathrm{Na}$ measured with a precision almost ten times better than ever before [35]. The spectrometer is also described in some detail and the results discussed in light of the shell quenching problem as well as comparisons to previous measurements.

\section{DESCRIPTION OF THE MISTRAL SPECTROMETER}

MISTRAL is a radio-frequency, transmission spectrometer based on the principle conceived and later realized by Smith
[36,37]. The Orsay version originally concentrated on the measurement of the antiproton mass but was also designed for mass measurements of exotic nuclides [38,39]. MISTRAL is currently installed at the ISOLDE mass separator facility at CERN [40]. ISOLDE furnishes low energy $(60 \mathrm{keV})$, high quality beams of radioactive nuclides produced by spallation, fission and/or fragmentation of thick targets bombarded by a $1 \mathrm{GeV}$ pulsed proton beam. With the MISTRAL spectrometer, the mass is determined via the cyclotron frequency $f_{c}$ of an ion of charge $q$, mass $m$, rotating in the magnetic field $B$

$$
f_{c}=\frac{q B}{2 \pi m} .
$$

\section{A. Static, mass-separation mode}

The lay-out of MISTRAL is shown in Fig. 1. The spectrometer consists mainly of a homogeneous magnetic field. The ion beam is transported through the stray field of the magnet and focused onto the entrance slit. Given a slight downward deflection, the ions follow a two-turn helicoidal trajectory (Fig. 1, inset center) whereupon they are extracted from the magnetic field and transported (using elements that are symmetric to the injection) to a secondary electron multiplier for counting. The transmission of the spectrometer (through the four $0.4 \mathrm{~mm} \times 5 \mathrm{~mm}$ slits that precisely define the nominal trajectory) is about $0.5 \%$ using the surface ionization reference source but can be lower than $0.01 \%$ using ISOLDE ion sources.

With an orbit diameter of $1000 \mathrm{~mm}$ and an entrance slit size of $0.4 \mathrm{~mm}$, a mass resolving power of 2500 is obtained. In order to obtain the high resolution needed for precision measurements, a radio-frequency modulation of the longitudinal kinetic energy is effected using two symmetric electrode structures (Fig. 1, inset right) located at the one-half and three-half turn positions inside the magnetic field. This way the ions make one cyclotron orbit between the two modulations. The radio-frequency voltage is applied to the common, central modulator electrode and the resulting trajectories are all isochronous. Depending on the phase of this voltage when the ions cross the gaps, the resulting longitudinal acceleration produces a larger or smaller cyclotron radius than that of the nominal trajectory. The ions are transmitted through the $0.4 \mathrm{~mm}$ exit slit when the net effect of the two modulations is zero. This happens when the radiofrequency voltage is an integer-plus-one-half multiple of the cyclotron frequency:

$$
f_{\mathrm{rf}}=\left(n+\frac{1}{2}\right) f_{c}
$$

which means that during the second modulation the ions feel exactly the opposite of what they felt during the first. The ion signal recorded over a wide radio-frequency scan shows narrow, transmission peaks that are evenly spaced at the cyclotron frequency (Fig. 1, inset left). The resolving power $R$ $=m / \Delta m$ will depend on the harmonic number $n$, the exit slit size $w$, and the modulation amplitude $D_{m}$ : 


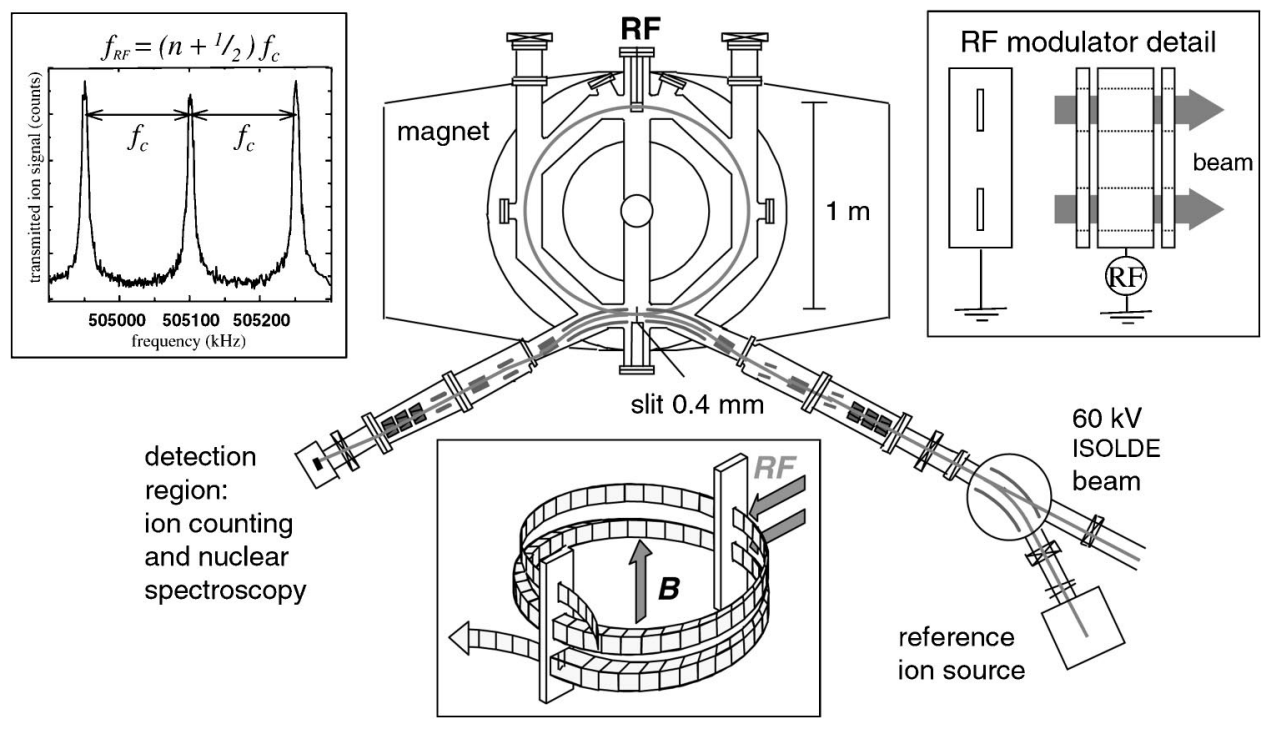

FIG. 1. Layout of the MISTRAL spectrometer (overhead view). The ion beams (coming from the right) are injected either from the ISOLDE beamline (at $60 \mathrm{keV}$ ) or from a reference ion source (variable energy). Inset (center) shows an isometric view of the trajectory envelope with the $0.4 \mathrm{~mm}$ injection slit followed by the first modulator at one-half turn, an opening to accommodate the modulated-ion trajectories at one turn, the second modulator at three-half turns, and then the exit slit. Inset (right) shows the modulator electrode structure, the geometry of which is selected for a given mass range (e.g., $20<A<70$ ). Inset (left) shows a transmitted ${ }^{39} \mathrm{~K}$ ion signal as a function of radio frequency spanning three harmonic numbers (about 3400). The mass resolution varies from 50000 to 100000 over the operational frequency range of $250-500 \mathrm{MHz}$ but can exceed 100000 by applying a higher radio-frequency voltage.

$$
R=2 \pi n \frac{D_{m}}{w} .
$$

A resolving power of $10^{5}$, for a $60 \mathrm{keV}{ }^{23} \mathrm{Na}$ ion beam having a cyclotron frequency of about $225 \mathrm{kHz}$, requires a $2.5 \mathrm{~mm}$ increase in the trajectory diameter (about $350 \mathrm{~V}$ of modulator voltage) and can already be achieved with a harmonic of only 1500 , corresponding to a radio frequency of about $340 \mathrm{MHz}$. Details on the calculation of the resolving power are given by Coc et al. [41]. The wings of the transmitted ion signal can be completely suppressed by using the "phase definition slit," located between the entrance and exit slits, to eliminate modulated ion trajectories with large radial excursions. The resulting peak shape is approximately triangular $[41,42]$. The phase-definition slit has a maximum opening of $5 \mathrm{~mm}$ and is reduced according to the radio-frequency power used (see Sec. II D and Fig. 3).

The modulator was designed to deliver up to approximately $500 \mathrm{~V}$ peak-to-peak over the frequency range of 250 to $500 \mathrm{MHz}$. A matching system using a quarter-wave transformer was designed with a variable length piston for tuning and having a $-3 \mathrm{~dB}$ bandwidth of about $2 \mathrm{MHz}$ (which corresponds to about eight harmonics of our ${ }^{23} \mathrm{Na}$ ion). A 1 $\mathrm{kW}$ amplifier, fed by a direct synthesizer with a frequency precision of $10^{-10}$, was custom built for this application as well as a radio-frequency circulator that transmits all the incident power to the modulator and sends any reflected power into a dissipative load in order to protect the amplifier.

\section{B. Dynamic, mass-measurement mode}

MISTRAL relies on the concurrent measurement of a (generally stable) reference nuclide whose mass is known with sufficient accuracy. There is at present no technique with which an absolute mass value may be measured directly with sufficient accuracy since this would require an extremely accurate measurement of the magnetic field directly along the ion trajectory.

The unknown mass is transmitted through the spectrometer alternately with a reference mass-without changing the magnetic field. Comparing masses in this way requires changing the transport energy of the reference beam and therefore the voltages of all electrostatic elements in the spectrometer (two quadrupole triplets, eight pairs of steering plates, and two cylindrical benders plus the injection switchyard bender). These comparisons are done in rapid succession (seconds) in order to eliminate error contributions due to the mean- and long-term drifts of the magnetic field.

Since the modulator matching system is tuned for a given frequency range, it is necessary to find the harmonic numbers $n_{r}$ and $n_{x}$ of the reference $m_{r}$ and unknown $m_{x}$ masses that correspond to approximately the same frequency (i.e., within the tuning pass band). The measurement of $m_{x}$ is thus obtained from the relation

$$
m_{x}=\frac{f_{r}}{f_{x}} \frac{\left(n_{x}+\frac{1}{2}\right)}{\left(n_{r}+\frac{1}{2}\right)} m_{r}
$$

where $f_{x}$ and $f_{r}$ are the unknown and reference frequency peak centroids, determined by a triangular fit (see Sec. II D). The harmonic numbers obey the relation

$$
n=\frac{2 \pi m f}{q B}-\frac{1}{2} .
$$


Since they are integers $\approx 2000$, a $10^{-4}$ accuracy is sufficient for $m$ and $B$ to determine them exactly. Even for unknown nuclides, $m$ is always predicted with at least such an accuracy. To measure $B$, a NMR probe is used. However, since this probe cannot be positioned on the nominal trajectory, the reading is corrected by recording peaks at several neighboring harmonics and measuring directly their spacing (Fig. 1, left inset). The resulting correction is $\sim 2 \times 10^{-4}$.

\section{Control system and data acquisition}

The experiment is controlled by a microprocessor working under a real-time kernel connected to a Sun/Unix workstation. After maximizing the transmission using the beam transport electrostatic elements and the magnetic field, the data acquisition mode is selected and frequency scan initiated. For stable nuclides, we make continuous scans of the radio frequency. Between scans, the beam transport element voltages are switched in proportion to the masses. Making frequent reference mass scans eliminates frequency shifts due to the long term drift of the magnetic field, and allows averaging over the short term.

In the case of short-lived nuclides (as well as elements with very rapid release times from the target matrix, such as $\mathrm{Na}$ ) there is insufficient time to scan the entire required frequency range after the impact of the proton pulse. In this case, a special acquisition mode is used (called, appropriately, point-by-point). For each radioactive beam pulse, the ion transmission signal is recorded for only one radiofrequency point (determined randomly) and the peak is reconstructed at the end. This mode not only allows one to increase statistics in the peak but for each point, the time dependence of the transmission can also be reconstructed. This offers an excellent way of identifying isobars. An example is given in Fig. 2 (top) for the isobaric doublet ${ }^{27} \mathrm{Na}-{ }^{27} \mathrm{Al}$. Since we count the ions in the beam that have been separated with very high resolution, we can produce very clean release curves as shown in Fig. 2 (middle). The stable $\mathrm{Al}$ peak is easily recognized by its constant release in Fig. 2 (bottom).

Furthermore, in case of variations in the ISOLDE production from pulse to pulse, it is possible to record the ion signal with the rf alternately on and off so that not just the intensity but the true transmission is measured in order to correctly normalize the peak. However, this procedure, used in run No. 1 (see Sec. III), was found to be not really necessary.

At ISOLDE, the protons are grouped into a supercycle of 12 pulses of up to $3 \times 10^{13}$ protons every $1.2 \mathrm{sec}$. The selection of these pulses coming to ISOLDE is variable, usually between 6 and 8 but with no particular regularity. When measuring the short-lived nuclides, we synchronize the experiment on this supercycle making a reference scan each time. We have found this sufficient to correct for the magnetic field drifts.

\section{Data analysis}

The primary aim of the data analysis is to determine the frequency corresponding to the maximum of transmission which is the center of symmetry of the peak. The fitting
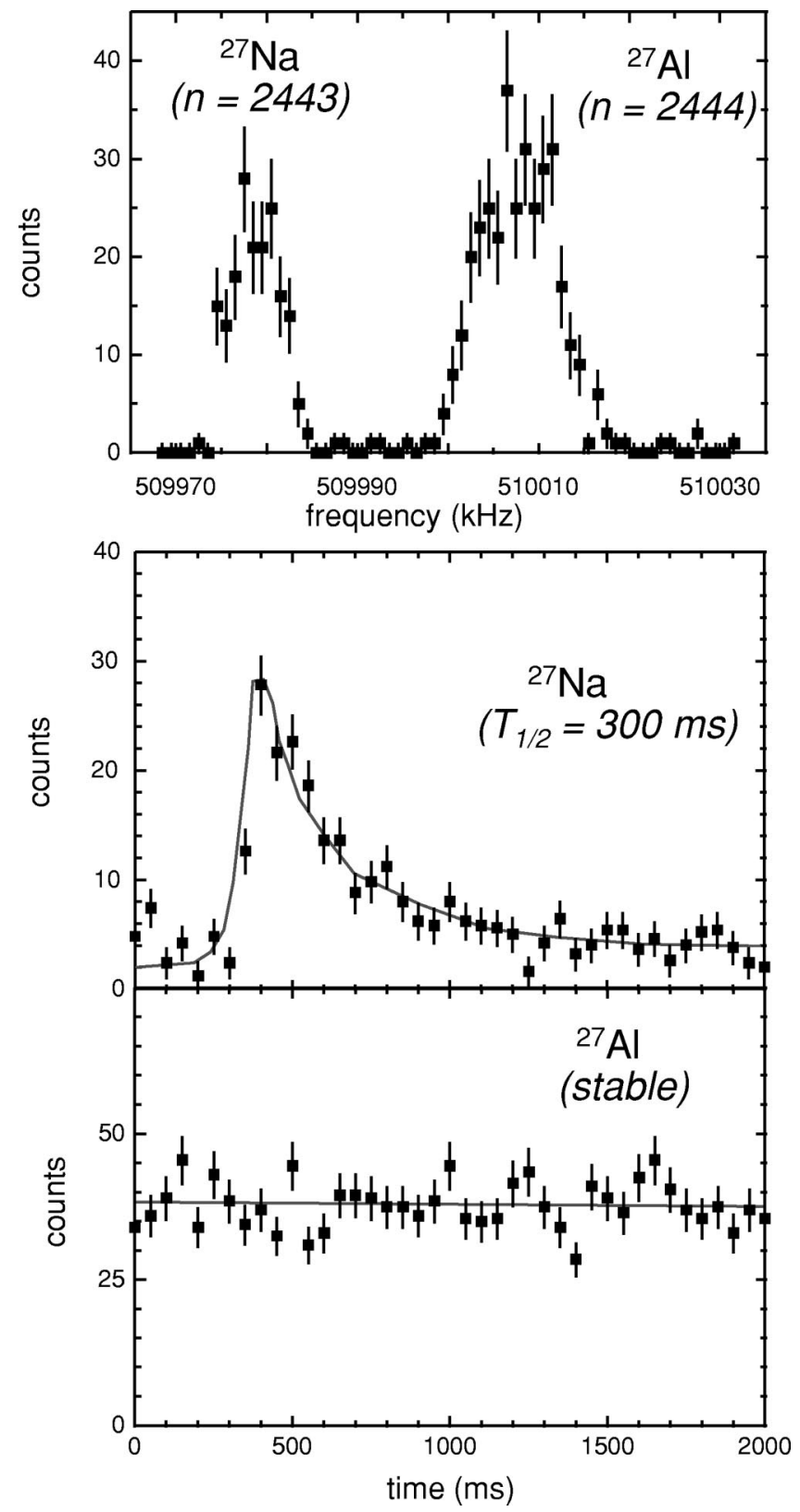

FIG. 2. Ion signal as a function of radio frequency for $A=27$ showing the two isobaric contributions of $\mathrm{Al}$ and $\mathrm{Na}$ (top). The peaks are well resolved but in cases where identification might be a problem we can examine the time projection of each peak which reveals either a fast release time indicating $\mathrm{Na}$ (middle) or a constant signal with time indicated the stable $\mathrm{Al}$ isobar (bottom).

procedure consists of iterating a least-squares adjustment of the measured yields $Y_{i}\left(f_{i}\right)$ using a triangular [41] function $g\left(f_{i}\right)$ depending on three parameters:

$$
g\left(f_{i}\right)=Y_{m}\left(1-\frac{\left|f_{i}-f_{m}\right|}{f_{w}}\right)
$$

where $f_{m}$ is the adjusted value of interest, $f_{w}$ is the frequency FWHM and $Y_{m}$ is the maximum yield (Fig. 3).

Wings, if not already suppressed by the phase-definition slit, are eliminated by selecting a restricted area of the peak. 


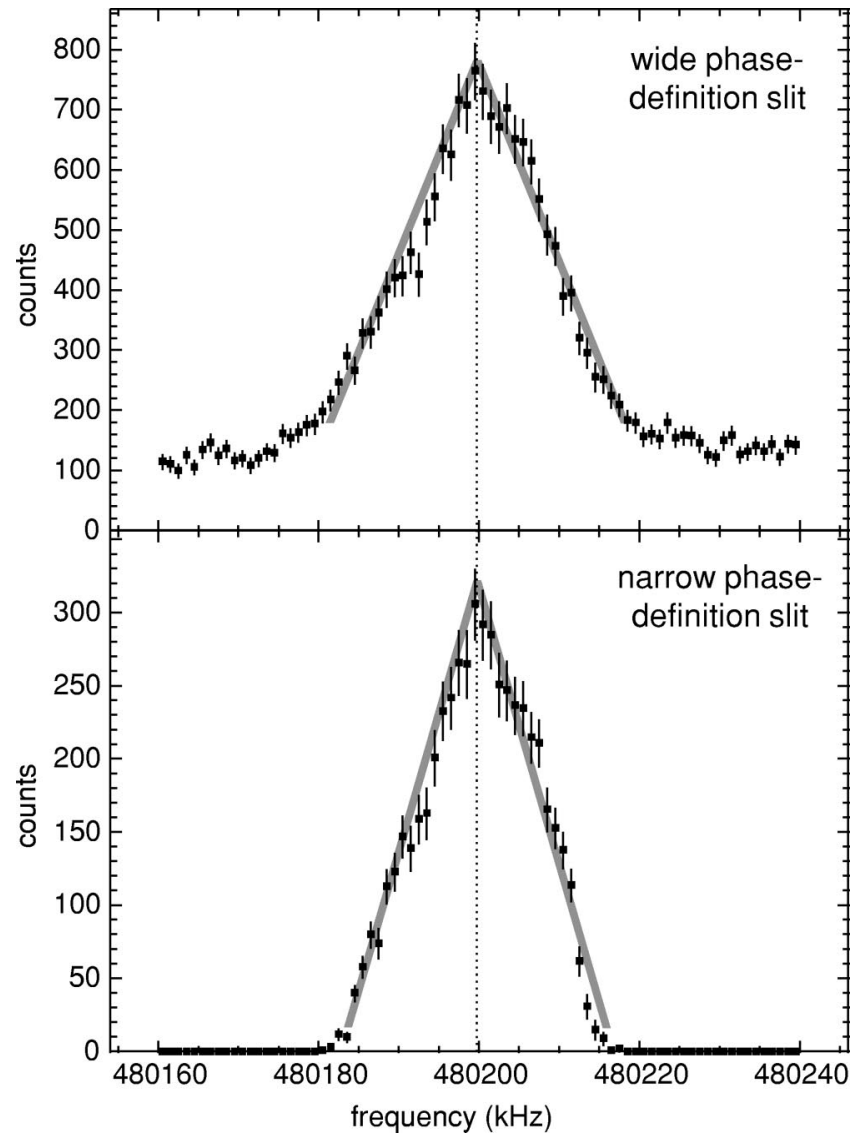

FIG. 3. Example of triangular fits of a transmitted ${ }^{24} \mathrm{Mg}$ ISOLDE mass peak performed (top) without phase limitation and (bottom) with a phase limitation that completely eliminates the wings. The resolving powers are (top) 19600 and (bottom) 28900 using only a moderate radio-frequency voltage.

The weight given to each point takes into account not only the statistics but also the short term fluctuations which have been determined experimentally:

$$
\begin{gathered}
\sigma^{2}\left(Y_{i}\right)=\left(\varepsilon_{Y} Y_{i}\right)^{2}+Y_{i}, \\
\sigma^{2}\left(f_{i}\right)=\left(\varepsilon_{B} f_{i}\right)^{2},
\end{gathered}
$$

where $\varepsilon_{Y}$ reflects the unstatistical fluctuations of the reference ion source intensity or of the ISOLDE production (if not normalized by a scan without RF) and $\varepsilon_{B}$ reflects the short term fluctuations of the magnetic field. Typically, $\varepsilon_{Y}$ $\sim 0.05$ and $\varepsilon_{B} \sim 5 \times 10^{-7}$.

This fitting procedure provides the frequency ratio $R_{f}$ $=f_{x} / f_{r}$ which is then converted into an atomic mass ratio $R_{m}=m_{x} / m_{r}$ using relation (4) and taking into account the electron mass and the relativistic correction for the ion velocity.

\section{RESULTS}

The data presented in this paper were recorded during two runs: run No. 1 in July 1998, and run No. 2 in November 1998. In both runs, the ISOLDE target made of uranium

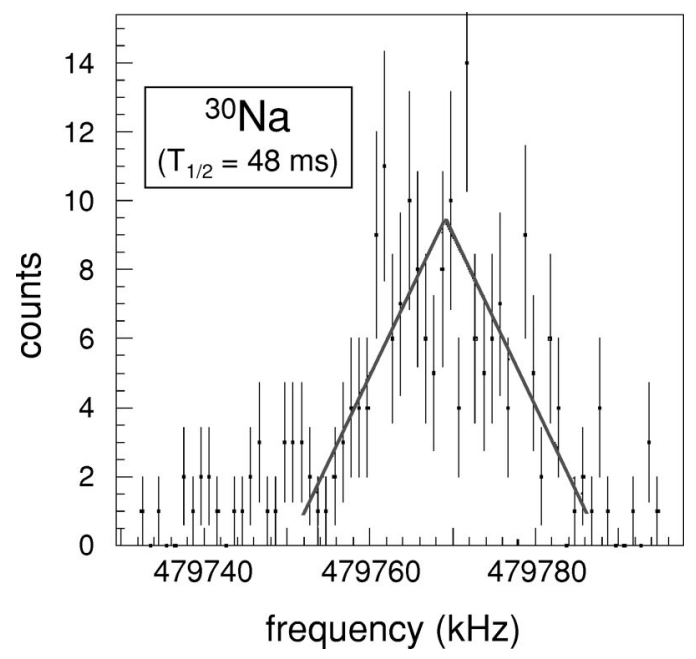

FIG. 4. Reconstructed peak for ${ }^{30} \mathrm{Na}\left(T_{1 / 2}=48 \mathrm{~ms}\right)$. This measurement is a sum of 25 series of 64 (random) frequency steps each recorded after the impact of one proton pulse on the ISOLDE target. The center frequency is derived from a triangular fit and corresponds to harmonic number 2421 of the cyclotron frequency in the $0.3868 \mathrm{~T}$ field. The mass resolution is about 25000 (reduced to favor transmission).

carbide was combined with a thermionic source which delivered relatively pure, singly ionized, radioactive alkali beams. The masses measured were ${ }^{23-30} \mathrm{Na}$ and ${ }^{27} \mathrm{Al}$. An example of a recorded mass peak is shown in Fig. 4 for ${ }^{30} \mathrm{Na}$.

During run No. 1, the high voltage of the MISTRAL source was limited to $V \leqslant 60 \mathrm{kV}$, constraining the reference mass to be heavier than the measured one. The reason is that, for a given magnetic field, the product $m \times V$ is fixed, and $V$ is always equal to $60 \mathrm{kV}$ for the measured mass from ISOLDE. Hence, ${ }^{39} \mathrm{~K}$ was the reference mass in all cases except those where ${ }^{23} \mathrm{Na}$ from the reference source was to be compared to ${ }^{23} \mathrm{Na}$ from ISOLDE. During run No. 2, this limitation was overcome and both ${ }^{23} \mathrm{Na}$ and ${ }^{39} \mathrm{~K}$ could be used as references for each mass from ISOLDE.

\section{A. Calibration procedure}

A comparison of the measured values to the ones taken from the AME'95 evaluation [1] for well-known masses revealed a dependency on the mass difference $m_{r}-m_{x}$ between the MISTRAL reference mass $m_{r}$ and the ISOLDE measured mass $m_{x}$ (Fig. 5). A calibration was thus necessary.

The relative mass difference between a measured value, $m_{x}$ and the tabulated one, $m_{x}^{0}$, is given by

$$
\Delta_{x}^{\text {meas }}=\frac{m_{x}-m_{x}^{0}}{m_{x}^{0}} .
$$

Note that the AME'95 value is used in $\Delta_{x}$ for convenience, since it only plays the role of a fulcrum and does not influence the final mass excess.

As the magnetic field is not sufficiently homogeneous over the entire volume seen by the ion beam, the nonzero value of $\Delta_{x}^{\text {meas }}$ is attributed to an imperfect superposition of the $m_{x}$ and the $m_{r}$ trajectories. This apparently linear devia- 

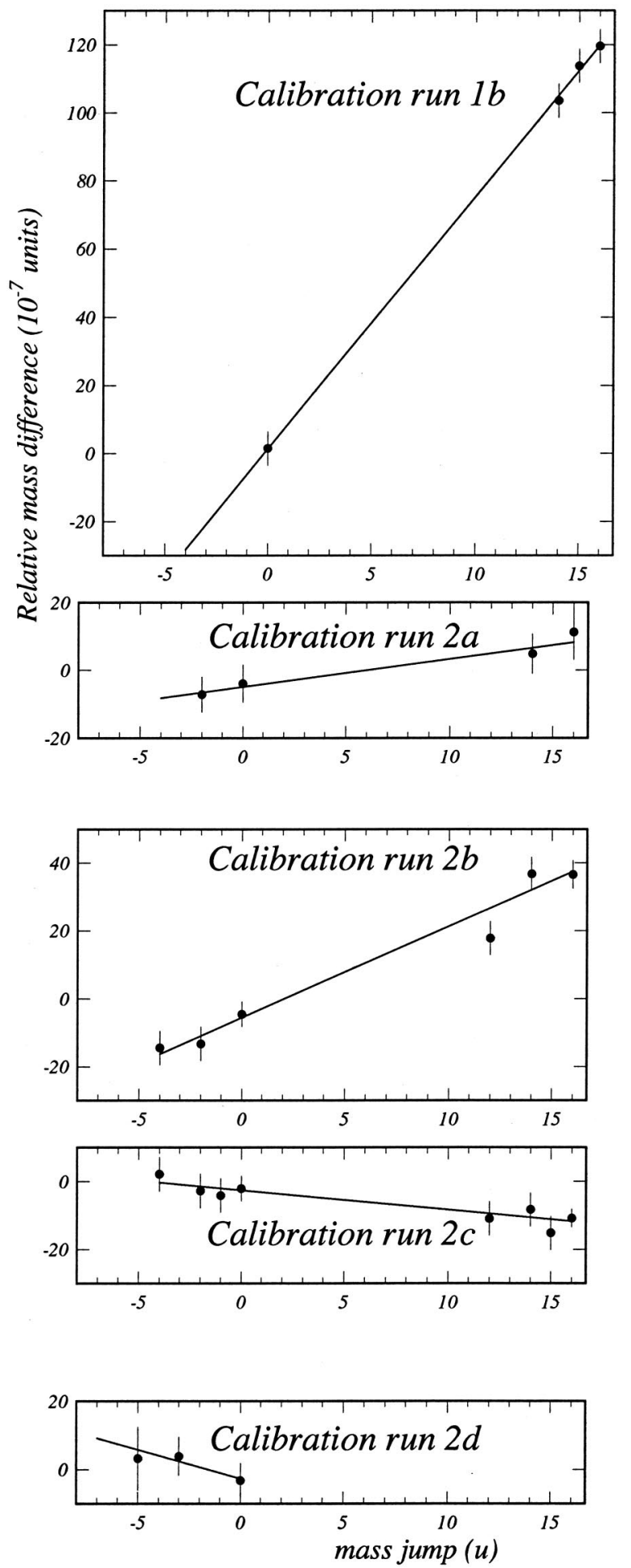

FIG. 5. Plot of the relative mass differences $\Delta_{x}^{\text {meas }}$, for the wellknown masses, with respect to the mass table AME'95, versus the mass jump $\left(m_{r}-m_{x}\right)$. The continuous lines represent the fits of the linear calibration function for each run.

tion has two possible components: (i) an insufficient overlap between the ISOLDE beam and the MISTRAL reference source beam positions which would produce a constant $\Delta_{x}^{\text {meas }}$ and (ii) a different injection angle caused by inadequately calibrated voltage changes that are necessary for transmission of the two beams of differing masses through the same magnetic field and which would produce $\Delta_{x}^{\text {meas }} \propto\left(m_{r}-m_{x}\right)$. This reasoning is based on measurements of the residual field gradient of the magnetic field. While higher order components are present, the linear gradients represent the largest contribution [43]. We have therefore adopted as a calibration function

$$
\Delta_{x}^{\text {meas }}=a\left(m_{r}-m_{x}\right)+b
$$

In order to check the validity of this calibration and to determine $a$ and $b$, we selected as "calibrants" the precisely known masses ${ }^{23-25} \mathrm{Na}$ and ${ }^{27} \mathrm{Al}$. A straight line was fitted to the corresponding $\Delta_{x}^{\text {meas }}$ values. To these points we add an additional calibration derived from the measurements of an unknown mass $x$ using the two reference masses ${ }^{23} \mathrm{Na}$ and ${ }^{39} \mathrm{~K}$. Two such measurements performed under exactly the same experimental conditions, yield

$$
\Delta_{x}^{\text {meas }}\left({ }^{39} \mathrm{~K}\right)-\Delta_{x}^{\text {meas }}\left({ }^{23} \mathrm{Na}\right)=[(39-x)-(23-x)] a=16 a .
$$

Taking into account the adjusted value for $b$ from the first set of calibrants, these values contributed to the averaged $\Delta_{x}^{\text {meas }}$ corresponding to $m_{r}-m_{x}=16$. The process was iterated to derive the final values for $a$ and $b$ and for their standard deviations.

While trying to fit the calibration function, it appeared that $b$ was fairly constant in the range of some $10^{-7}$. On the contrary, $a$ changed every time a tuning of the injection optics of the ISOLDE or MISTRAL beams was necessary. Therefore, each of the two runs was divided into subsets corresponding to beam optics settings: $1 \mathrm{a}, 1 \mathrm{~b}, 2 \mathrm{a}, 2 \mathrm{~b}, 2 \mathrm{c}$, and $2 \mathrm{~d}$ (see Table I).

The complete set of measured relative mass differences and calibrations for both runs is presented (in chronological order) in Table I. The values of $\Delta_{x}^{\text {meas }}$ for each mass are given using the corresponding references: ${ }^{23} \mathrm{Na}$ in column 3 and ${ }^{39} \mathrm{~K}$ in column 4 . The errors given in Table I are statistical plus the intensity and magnetic field fluctuation errors (see Sec. II D).

In the fitting procedure of the calibration, it appeared that the $\chi^{2}$ values were much too large (up to $\chi^{2}=20$ ), revealing the existence of a systematic error. A good consistency was obtained by adding quadratically a systematic uncertainty of $5 \times 10^{-7}$ to each measurement. The resulting calibrations for runs No. 1b, 2a, 2b, 2c, and 2d are shown in Fig. 5, and the corresponding values for the calibration parameters $a$ and $b$ are reported in Table II.

In the case of run No. 1a, only ${ }^{27} \mathrm{Al}$ was available as a calibrant. Therefore, $b$ was assumed to have the value determined in run No. $1 b$. In the case of run No. $2 d$, due to the lack of calibrant masses, the calibration was determined using the evaluated masses for ${ }^{26} \mathrm{Na}$ and ${ }^{28} \mathrm{Na}$ as obtained in Sec. IV A. 
TABLE I. Experimental results, expressed as relative mass differences $\Delta_{x}^{\text {meas }}$, in chronological order. In column 2, the mention "calib." indicates a calibrant mass in a given run subset; an asterisk indicates that ${ }^{27} \mathrm{Na}$ could not be measured because it was not sufficiently mass separated from ${ }^{27} \mathrm{Al}$. In columns 3-4 are the $\Delta_{x}^{\text {meas }}$ measured values and their statistical plus fluctuations errors between parentheses.

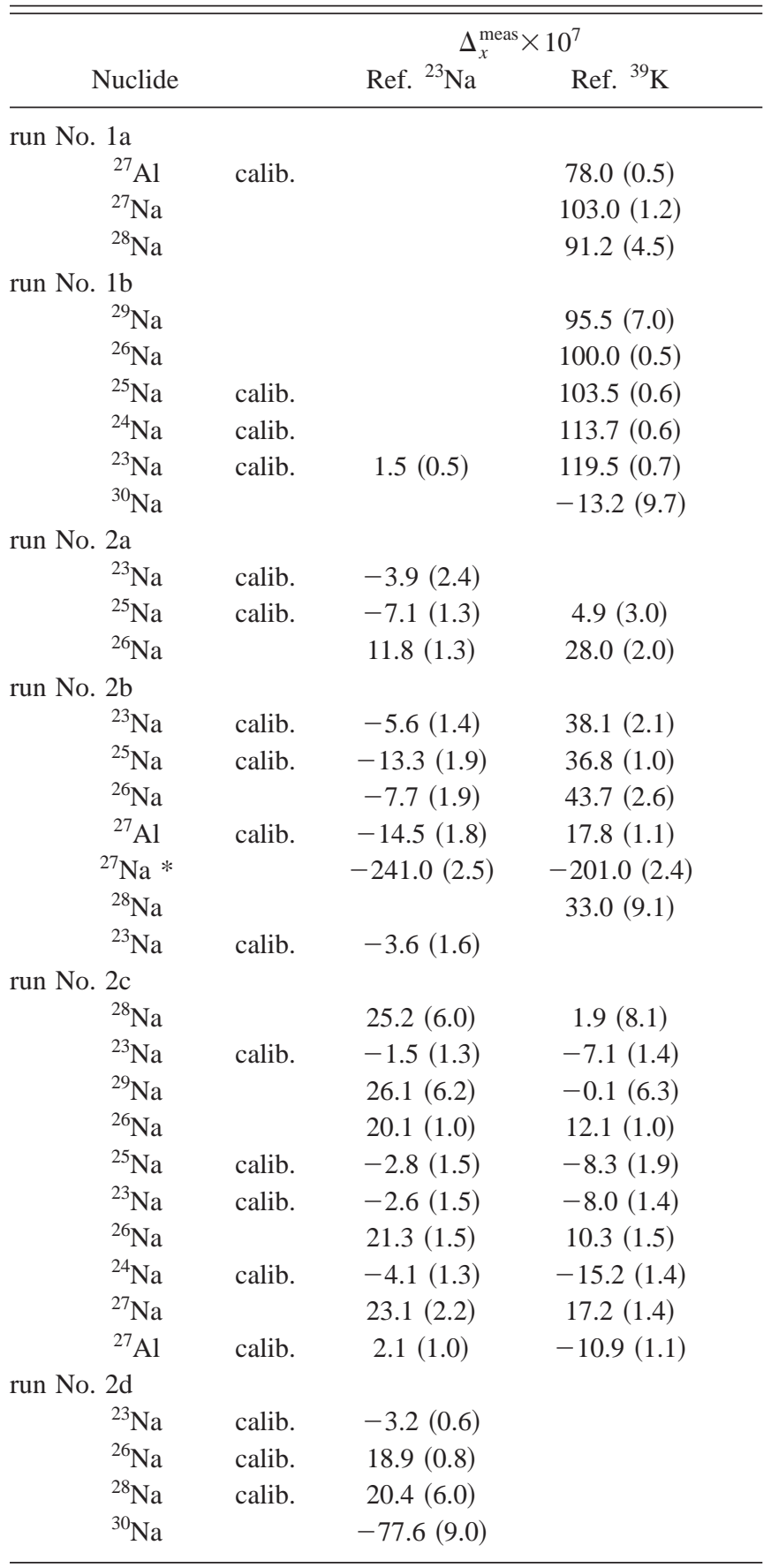

\section{B. Results after calibration}

The values of $\Delta_{x}^{\text {meas }}$ have been corrected using the calibration

$$
\Delta_{x}^{\text {corr }}=\Delta_{x}^{\text {meas }}-a\left(m_{r}-m_{x}\right)-b .
$$

The resulting values are presented in Table III.
TABLE II. Calibration parameters used for each of the measurement periods, after consideration of the systematic error of 5 $\times 10^{-7}$ (see text). In column 4 , the correlation coefficients between the fitted parameters $a$ and $b$ are given. The $\chi$ values of the calibration fits are given in column 5 .

\begin{tabular}{lcccc}
\hline \hline Run & $\begin{array}{c}\text { Slope } \\
\left(a \times 10^{7}\right)\end{array}$ & $\begin{array}{c}\text { Offset } \\
\left(b \times 10^{7}\right)\end{array}$ & Correl. & $\chi$ \\
\hline No. 1a & $6.4(0.6)$ & & & \\
No. 1b & $7.4(0.4)$ & $1.4(5.0)$ & -0.86 & 0.3 \\
No. 2a & $0.82(0.38)$ & $-4.9(3.5)$ & -0.55 & 0.4 \\
No. 2b & $2.69(0.23)$ & $-5.6(2.4)$ & -0.62 & 1.0 \\
No. 2c & $-0.57(0.18)$ & $-2.6(2.1)$ & -0.69 & 0.5 \\
No. 2d & $-1.7(1.8)$ & $-2.7(4.8)$ & +0.70 & 0.4 \\
\hline \hline
\end{tabular}

\section{Isobaric comparisons}

The comparison of the results for two isobars produced by ISOLDE, obtained in two successive measurements, lead to null values for $a$ and $b$ parameters (same beam, no mass jump) as in the case of the isobaric doublet ${ }^{27} \mathrm{Na}-{ }^{27} \mathrm{Al}$. Four measurements were made using this doublet (one of which does not appear in Table I due to lack of calibration data). The results are given in Table IV. In this case adding a 2 $\times 10^{-7}$ systematic uncertainty provided consistency for the four values $(\chi \simeq 1)$.

\section{Final results}

The measured $\Delta_{x}^{\text {corr }}$ values obtained for each nuclide in Table III have been averaged. In Table V, the results concerning the calibrant masses are listed. As displayed in column 4, the agreement with the AME'95 mass table is excellent, confirming the validity of the method used and of the chosen calibration relation.

In Table VI, the results concerning the masses measured by MISTRAL are reported.

The last line of Table VI shows that the determination of the ${ }^{27} \mathrm{Na}$ mass by "isobaric comparison" is indeed more precise, by a factor of almost 3, than the "standard method." The values obtained through the two methods agree very well, but the three measurements used in the "standard method" are also used in the isobaric doublet evaluation. Therefore the two results given in Table VI for ${ }^{27} \mathrm{Na}$ should not be considered as independent. Since the "standard method" was more thoroughly checked, only its result will be retained for further discussion.

The agreement between the different measurements from runs Nos. 1 and 2 is quite reasonable. The overall dispersion of the measurements compared to their mean values, shown in Fig. 6, is quite good, corresponding to $\chi=0.94$.

\section{DISCUSSION OF THE RESULTS}

\section{A. Evaluation}

The MISTRAL results are compared in Fig. 7 to the accepted mass values from the 1995 "Atomic Mass Evaluation" (AME'95) [1]. There is a very good agreement for the precisely known masses of ${ }^{23-25} \mathrm{Na}$ and ${ }^{27} \mathrm{Al}$. The average 
TABLE III. Experimental results, after correction using the calibration, expressed as relative mass differences $\Delta_{x}^{\text {corr }}$. In column 2, "calib" indicates a calibrant mass.

\begin{tabular}{|c|c|c|c|}
\hline \multirow[b]{2}{*}{ Nuclide } & & \multicolumn{2}{|c|}{$\Delta_{x}^{\text {corr }} \times 10^{7}$} \\
\hline & & Ref. ${ }^{23} \mathrm{Na}$ & Ref. ${ }^{39} \mathrm{~K}$ \\
\hline \multicolumn{4}{|l|}{ run No. 1a } \\
\hline${ }^{27} \mathrm{Al}$ & calib. & & $-0.2(10.1)$ \\
\hline${ }^{27} \mathrm{Na}$ & & & $24.8(10.2)$ \\
\hline${ }^{28} \mathrm{Na}$ & & & $19.4(10.7)$ \\
\hline \multicolumn{4}{|l|}{ run No. 1b } \\
\hline${ }^{29} \mathrm{Na}$ & & & $20.1(9.0)$ \\
\hline${ }^{26} \mathrm{Na}$ & & & $2.4(5.7)$ \\
\hline${ }^{25} \mathrm{Na}$ & calib. & & $-1.5(5.8)$ \\
\hline${ }^{24} \mathrm{Na}$ & calib. & & $1.3(5.9)$ \\
\hline${ }^{23} \mathrm{Na}$ & calib. & $0.1(7.1)$ & $-0.3(6.0)$ \\
\hline${ }^{30} \mathrm{Na}$ & & & $-81.2(11.2)$ \\
\hline \multicolumn{4}{|l|}{ run No. $2 \mathrm{a}$} \\
\hline${ }^{23} \mathrm{Na}$ & calib. & $1.0(6.6)$ & \\
\hline${ }^{25} \mathrm{Na}$ & calib. & $-0.6(6.5)$ & $-1.7(7.4)$ \\
\hline${ }^{26} \mathrm{Na}$ & & $19.2(6.7)$ & $22.2(6.8)$ \\
\hline \multicolumn{4}{|l|}{ run No. $2 b$} \\
\hline${ }^{23} \mathrm{Na}$ & calib. & $0.0(5.7)$ & $1.9(6.1)$ \\
\hline${ }^{25} \mathrm{Na}$ & calib. & $-2.5(6.0)$ & $5.9(5.7)$ \\
\hline${ }^{26} \mathrm{Na}$ & & $5.7(6.1)$ & $15.4(6.1)$ \\
\hline${ }^{27} \mathrm{Al}$ & calib & $1.5(6.1)$ & $-7.9(5.6)$ \\
\hline${ }^{28} \mathrm{Na}$ & & & 9.9 (10.6) \\
\hline${ }^{23} \mathrm{Na}$ & calib. & $2.0(5.8)$ & \\
\hline \multicolumn{4}{|l|}{ run No. 2c } \\
\hline${ }^{28} \mathrm{Na}$ & & $25.0(8.3)$ & $10.8(9.7)$ \\
\hline${ }^{23} \mathrm{Na}$ & calib. & $1.1(5.6)$ & $4.6(5.6)$ \\
\hline${ }^{29} \mathrm{Na}$ & & $25.3(8.5)$ & $8.2(8.2)$ \\
\hline${ }^{26} \mathrm{Na}$ & & $21.0(5.7)$ & $22.1(5.4)$ \\
\hline${ }^{25} \mathrm{Na}$ & calib. & $-1.3(5.7)$ & $2.3(5.7)$ \\
\hline${ }^{23} \mathrm{Na}$ & calib. & $0.0(5.6)$ & $3.7(5.6)$ \\
\hline${ }^{26} \mathrm{Na}$ & & $22.2(5.8)$ & $20.3(5.5)$ \\
\hline${ }^{24} \mathrm{Na}$ & calib. & $-2.1(5.6)$ & $-4.0(5.5)$ \\
\hline${ }^{27} \mathrm{Na}$ & & $23.4(6.1)$ & $26.6(5.5)$ \\
\hline${ }^{27} \mathrm{Al}$ & calib. & $2.4(5.7)$ & $-1.5(5.4)$ \\
\hline \multicolumn{4}{|l|}{ run No. 2d } \\
\hline${ }^{23} \mathrm{Na}$ & calib. & $-0.5(7.0)$ & \\
\hline${ }^{26} \mathrm{Na}$ & calib & $16.5(6.4)$ & \\
\hline${ }^{28} \mathrm{Na}$ & calib & $14.6(10.2)$ & \\
\hline${ }^{30} \mathrm{Na}$ & & $-86.8(14.2)$ & \\
\hline
\end{tabular}

standard deviation (difference in masses divided by the experimental uncertainty) for these four reference masses is extremely good (0.52) showing that the quoted precisions (ranging from 2 to $9 \times 10^{-7}$ for the measured masses) are certainly not overestimated. This gives confidence to the estimated systematic errors and to the calibration of the observed frequency shifts, even though their origin is not yet fully understood. Furthermore, the masses of ${ }^{23-30} \mathrm{Na}$ were measured during two separate data taking periods yielding consistent results.

The MISTRAL measurement for the ${ }^{26} \mathrm{Na}$ mass differs significantly from the AME'95 value. In fact, the nine individual
TABLE IV. Measured values of $\Delta_{x}^{\text {meas }} \times 10^{7}$ obtained for ${ }^{27} \mathrm{Na}$ and ${ }^{27} \mathrm{Al}$ both coming from the same source. Their difference gives directly the true value of $\Delta_{x}$ for ${ }^{27} \mathrm{Na}$ using no calibration. The first measurement was not included in Table I due to lack of calibration data. The errors indicated between parentheses are statistical plus fluctuations ones. A systematic error of $2 \times 10^{-7}$ is added (quadratically) to each of the ${ }^{27} \mathrm{Na}-{ }^{27} \mathrm{Al}$ results.

\begin{tabular}{lcccc}
\hline \hline Run & Reference & ${ }^{27} \mathrm{Na}$ & ${ }^{27} \mathrm{Al}$ & ${ }^{27} \mathrm{Na}-{ }^{27} \mathrm{Al}$ \\
\hline No. 1 & ${ }^{39} \mathrm{~K}$ & $96.2(5.0)$ & $69.8(2.0)$ & $26.4(5.5)$ \\
No. 1a & ${ }^{39} \mathrm{~K}$ & $103.0(1.2)$ & $78.0(1.2)$ & $25.0(1.3)$ \\
No. 2c & ${ }^{23} \mathrm{Na}$ & $23.1(2.2)$ & $2.1(1.0)$ & $21.0(2.4)$ \\
No. 2c & ${ }^{39} \mathrm{~K}$ & $17.2(1.4)$ & $-10.9(1.1)$ & $28.1(1.8)$ \\
\hline \hline
\end{tabular}

measurements for this nuclide (Fig. 6) are all higher, with an average difference of $40 \pm 7 \mathrm{keV}$.

A new mass evaluation would consider, among the six publications determining the mass of ${ }^{26} \mathrm{Na}$ (see Fig. 8), only two significant data (the insignificant ones are those with 10 times less weight than the combination of all other accepted data, following AME'93 [44], p. 200): (i) the $\left(t,{ }^{3} \mathrm{He}\right)$ reaction energy of Flynn and Garrett [45] yielding a mass excess value of $-7411.7(21.5) \mathrm{u}$ nucleon for ${ }^{26} \mathrm{Na}$ and (ii) the MISTRAL one giving -7366.7(6.8) u nucleon, where the error bar has been multiplied by the $\chi$ value (see Table VI). These two data are in disagreement, with $\chi=2.0$ for their average. However, such an average is adopted, without scaling by $\chi$, following the policies of the mass evaluation [1], and yields for ${ }^{26} \mathrm{Na}$ a mass value of $-7370.8(6.5) \mathrm{u}$ nucleon. This value has been used in the calibration of run No. $2 \mathrm{~d}$ (see Sec. III A) where one of the two measurements of the mass of ${ }^{30} \mathrm{Na}$ has been performed.

The result for the mass of ${ }^{27} \mathrm{Na}$ is $63 \mathrm{keV}$ higher than in the 1995 mass table. The latter value was mainly determined from the average of two ${ }^{26} \mathrm{Mg}\left({ }^{18} \mathrm{O},{ }^{17} \mathrm{~F}\right)$ reaction energy data $[48,49]$. The mass we derive agrees perfectly with the measurement of Fifield et al. [48], but strongly disagrees with the result of the Munich group [49]. Examination of the two papers shows that the former has 50 times higher statistics and a more correct peak shape. Therefore its agreement with the present result gives confidence in our mass for ${ }^{27} \mathrm{Na}$.

The MISTRAL masses for ${ }^{28} \mathrm{Na}$ and ${ }^{29} \mathrm{Na}$ are in agreement with-but much more precise than-the AME'95 ones. Our knowledge for these masses is thus improved by a factor five. For both masses we are left with only one significant datum: the one from the present work. This value of ${ }^{28} \mathrm{Na}$ has been used in the calibration of run No. $2 \mathrm{~d}$ (see Sec. III A).

TABLE V. Averaged relative deviations from the AME'95 mass table for the calibrant nuclides ( $n$ is the number of measurements).

\begin{tabular}{cccc}
\hline \hline Nuclide & $n$ & $\chi$ & $\Delta_{x}^{\text {corr }} \times 10^{7}$ \\
\hline${ }^{23} \mathrm{Na}$ & 9 & 0.3 & $1.5(1.9)$ \\
${ }^{24} \mathrm{Na}$ & 3 & 0.5 & $-1.7(3.3)$ \\
${ }^{25} \mathrm{Na}$ & 5 & 0.6 & $0.7(2.6)$ \\
${ }^{27} \mathrm{Al}$ & 5 & 0.7 & $-1.4(2.7)$ \\
\hline \hline
\end{tabular}


TABLE VI. MISTRAL new measurements. In columns 4, 5: relative $\left(\Delta_{x}^{\text {corr }}\right)$ and absolute $\left(\delta m_{x}=m_{x}\right.$ $-m_{x}^{0}$ ) deviations from the AME'95 mass table. In column 6: final MISTRAL mass excesses. To be conservative, the uncertainty in this last column, which expresses our final values, has been multiplied by $\chi$ in the case of ${ }^{26} \mathrm{Na}$.

\begin{tabular}{lccccc}
\hline \hline Nuclide & $n$ & $\chi$ & $\Delta_{x}^{\text {corr }} \times 10^{7}$ & $\delta m_{x}$ (u nucleon) & Mass excess (u nucleon) \\
\hline${ }^{26} \mathrm{Na}$ & 9 & 1.3 & $16.7(2.0)$ & $43(5)$ & $-7367(7)$ \\
${ }^{27} \mathrm{Na}$ & 3 & 0.3 & $25.1(3.8)$ & $68(11)$ & $-5922(11)$ \\
${ }^{28} \mathrm{Na}$ & 4 & 0.8 & $17.2(4.8)$ & $48(14)$ & $-1062(14)$ \\
${ }^{29} \mathrm{Na}$ & 3 & 1.0 & $17.5(4.9)$ & $51(14)$ & $2861(14)$ \\
${ }^{30} \mathrm{Na}$ & 2 & 0.3 & $-83.3(8.8)$ & $-250(27)$ & $8976(27)$ \\
Isobaric method & & & & & $-5922(4)$ \\
${ }^{27} \mathrm{Na}$ & 4 & 1.0 & $25.2(1.5)$ & $68(4)$ & \\
\hline \hline
\end{tabular}

Finally, the mass we derive for ${ }^{30} \mathrm{Na}$ strongly disagrees with the mass table. A closer examination locates the disagreement (see Fig. 9) to only one experiment [7] in which the mass of ${ }^{30} \mathrm{Na}$ was derived from a time-of-flight (TOF) measurement at LAMPF. This datum, given with a higher precision than all the older ones, weighed dominantly in the AME'95 adjustment. One can notice in Fig. 9 that this discrepant result superseded an earlier TOF measurement of the same group [4] which is also at strong variance with our result, but in the opposite direction. In the most recent of these two publications, Zhou et al. [7] explained the contradictory results by an isobaric contamination of ${ }^{30} \mathrm{Na}$ by ${ }^{30} \mathrm{Mg}$ in the earlier work. Figure 9 shows that the MISTRAL result agrees nicely with all other data, but is at least one order of magnitude more precise.

\section{B. Comparison to mass models}

The list of microscopic binding energy predictions is fairly restrained. Apart from the early Hartree-Fock calcula-

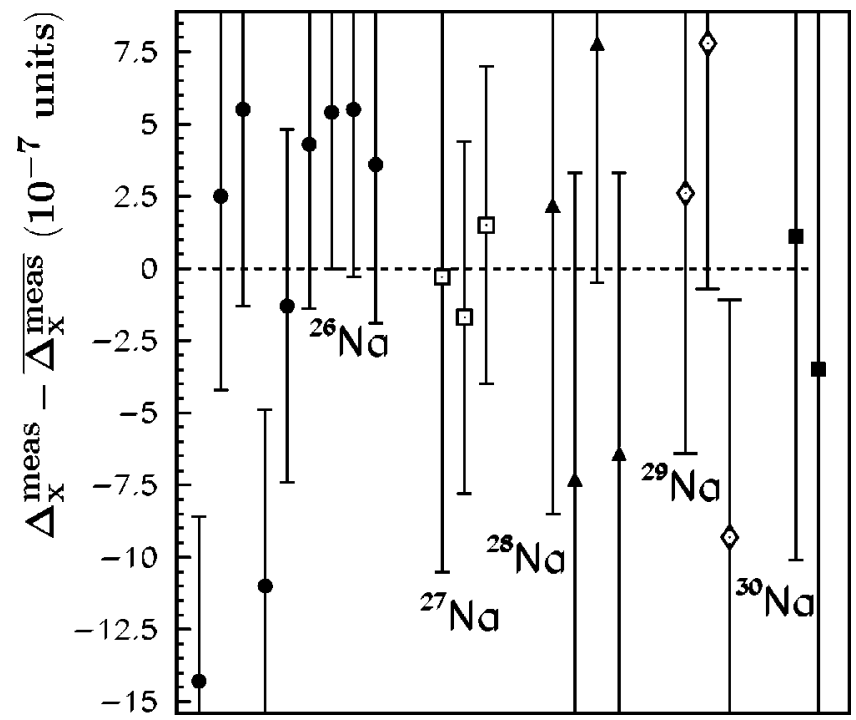

FIG. 6. Comparison of the individual measurements, for all the masses determined in this work, with their mean value: ${ }^{26} \mathrm{Na}$, full circles; ${ }^{27} \mathrm{Na}$, open squares; ${ }^{28} \mathrm{Na}$, full triangles; ${ }^{29} \mathrm{Na}$, open diamonds; and ${ }^{30} \mathrm{Na}$, full squares. tions of Campi et al. [15], where strong deformation, stabilized by rotational energy, was considered, and the shell model work of Warburton, Becker, and Brown [19], "modern," microscopic methods (e.g., Refs. [51,52]) have yet to produce calculations for odd- $Z$ nuclides. An exception is the work of Caurier et al. [21], and very recently, a complete mass table based on Hartree-Fock BCS constructed by Goriely, Tondeur, and Pearson [50]. This shortcoming, and the fact that microscopic models, for all their intense computing power requirements, have done only a moderate job in calculating the total binding energy, has promoted the development of mass models and mass formulas based on either approximations, e.g., the extended Thomas-Fermi Strutinski integral (ETFSI) [53] or macroscopic-microscopic formulations, e.g., the finite-range droplet model (FRDM) [54], that adjust their (sometimes numerous) parameters to measured masses.

The resulting MISTRAL mass of ${ }^{30} \mathrm{Na}$ confirms and even slightly enhances the overbinding of $\mathrm{Na}$ isotopes at $N=19$ as shown in Fig. 10. This tendency contradicts even more $N$ $=20$ being a magic number at $Z=11$ as it is at higher $Z$ [see the $Z=15(\mathrm{P})$ and $Z=16(\mathrm{~S})$ curves in Fig. 10] and reinforces the strength of the deformation starting at $N=19$. The higher binding energy measured in this work pulls the mass surface closer to the Hartree-Fock predictions of both Campi

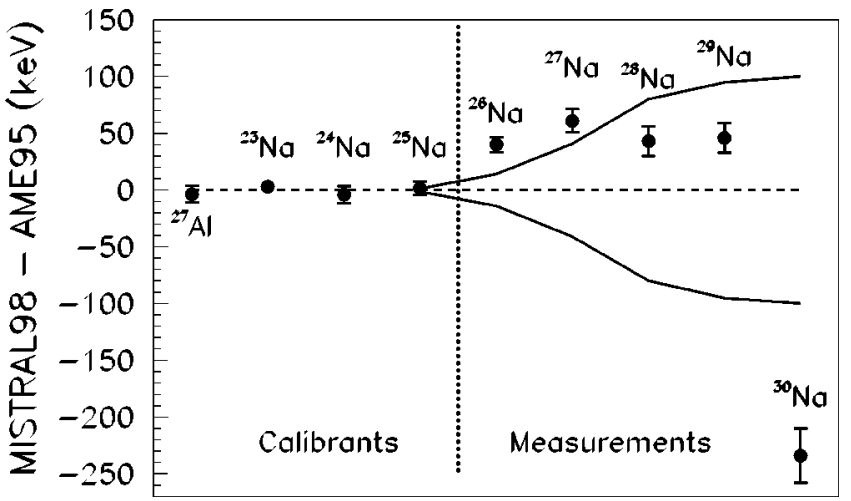

FIG. 7. The MISTRAL results compared to the AME'95 for ${ }^{27} \mathrm{Al}$ and for the sodium isotopes from $A=23$ to 30 . The zero line represents the values from the mass table and the two continuous symmetrical lines represent the table uncertainties. 


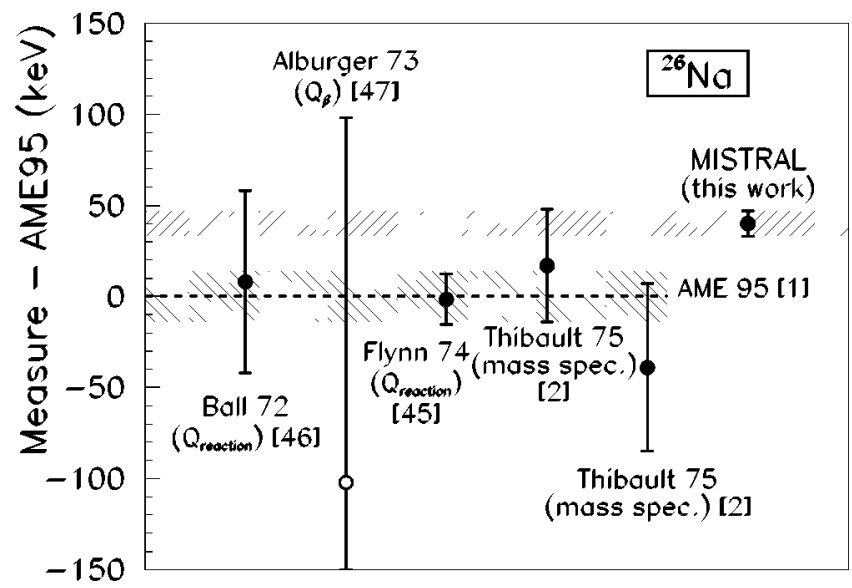

FIG. 8. Comparison of the results of all experiments in which the mass of ${ }^{26} \mathrm{Na}$ has been determined. The dashed area represents the $1 \sigma$ limit of the AME'95 value [1], based on the four data represented by full symbols which were obtained by (left to right) $\left({ }^{7} \mathrm{Li},{ }^{7} \mathrm{Be}\right)$ reaction energy [46]; $\beta$ end-point energy [47]; $\left(t,{ }^{3} \mathrm{He}\right)$ reaction energy [45]; mass spectrometry [2] by the Orsay group at CERN; and by rf mass spectrometry (this work).

et al. [15] and HFBCS-1 [50]. The shell model predictions [21] on the other hand, miss the effect at $N=19$ and predict the onset of deformation one neutron further. This is perhaps due to the uncertainty, mentioned in Ref. [21], of whether the intruder orbits responsible for this shell "opening" effect are dominated by $2 p-2 h$ or $4 p-4 h$ excitations. The latter possiblity was not explored in their work due to the great increase this incurs in valence space and hence, computation requirements.

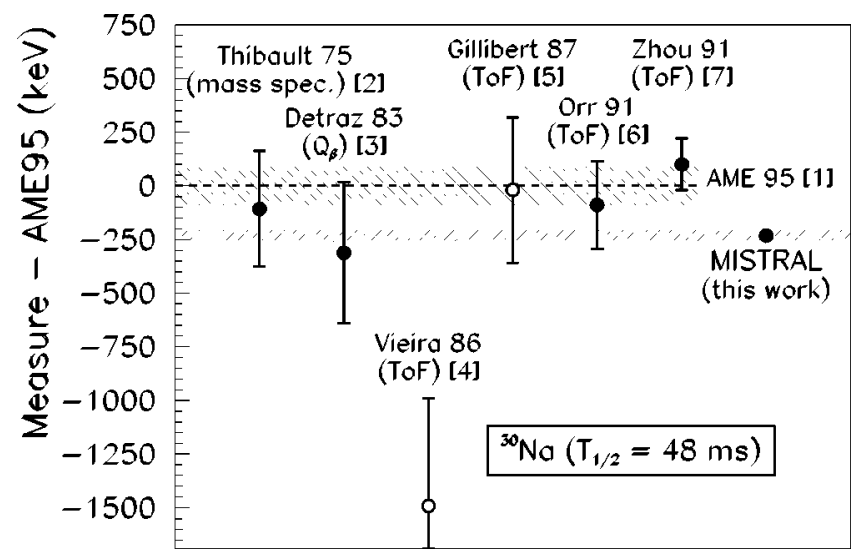

FIG. 9. Comparison of the results of all experiments in which the mass of ${ }^{30} \mathrm{Na}$ has been determined. The dashed area represents the $1 \sigma$ limit of the AME'95 value, based on the four data represented by full symbols. The two data with open symbols were superseded by more recent measurements of the same groups. The measurements represented here were obtained by (left to right) mass spectrometry [2] and $\beta$ end-point energy [3] by the Orsay group at CERN; four time-of-flight (TOF) measurements by two groups at LAMPF [4], GANIL [5], GANIL [6], and LAMPF [7]; and by rf mass spectrometry (this work).

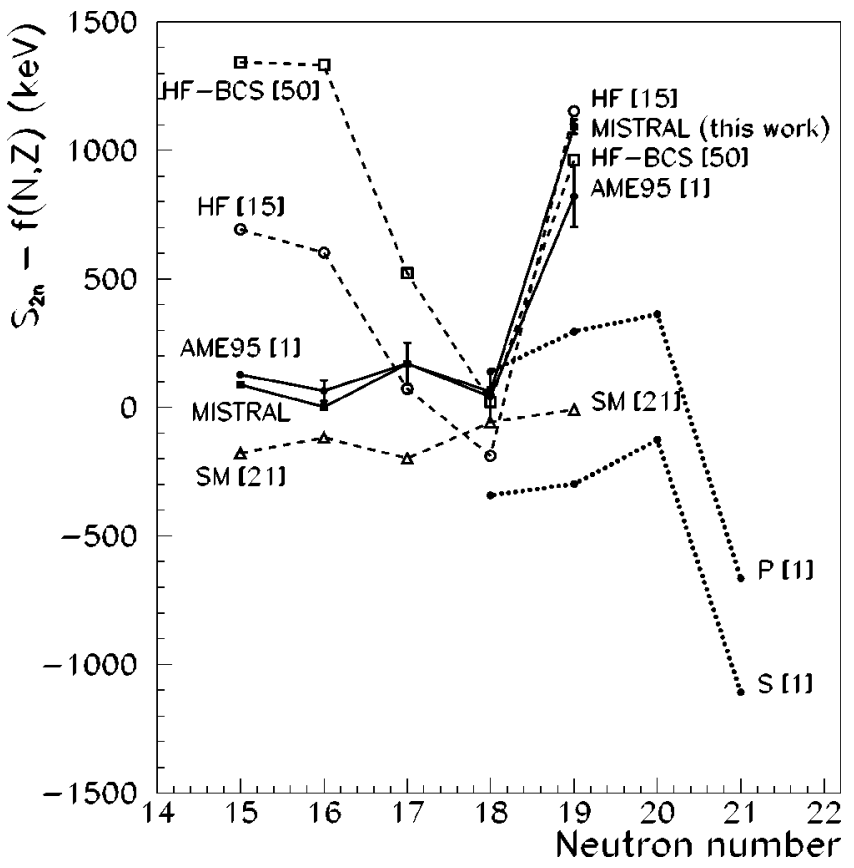

FIG. 10. Two-neutron separation energies $S_{2 n}$ plotted as a function of neutron number for the neutron-rich sodium isotopes $(Z$ =11): MISTRAL measurements (filled squares), the AME'95 [1] (filled circles), Hartree-Fock predictions of Campi et al. [15] (open circles), the very recent Hartree-Fock mass table HFBCS-1 of Goriely, Tondeur, and Pearson [50] (open squares), and shell model predictions of Caurier et al. [21] (open triangles). To illustrate the fine structure of these curves, the following empirical function of $N$ and $Z$ has been subtracted: $f=-3300 N+100 N Z+700 Z+39800$. The lines corresponding to $Z=15$, phosphorus $(\mathrm{P})$ and $Z=16$, sulfur $(S)$ from the AME'95 [1] are included to show the tendency observed where $N=20$ can still be considered magic.

\section{CONCLUSION}

We have presented results for the masses of neutron-rich $\mathrm{Na}$ isotopes using a new technology for mass measurements that is especially suitable for very short-lived species. These mass measurements, exploiting a radio-frequency modulation technique, are accurate to the precision that was originally aimed for: $14 \mathrm{keV}$, or $5 \times 10^{-7}$ for the shortest-lived measured nuclide, ${ }^{28} \mathrm{Na}(30.5 \mathrm{~ms})$. The method also has a reasonable sensitivity limit of about $10^{3}$ ions per pulse delivered by the on-line mass separator ISOLDE at CERN.

The results represent the most accurate measurements to date for the short-lived nuclides ${ }^{28-30} \mathrm{Na}$ which lie in the so-called "island of inversion" around the $N=20$ shell closure. The measurements not only confirm the previous values but even show an enhancement of the binding energy which further argues for the erosion of the normally stabilizing "magic" number shell effect.

These results are the first fruit of the commissioning of the MISTRAL program at ISOLDE. Efforts are currently underway to eliminate the systematic error using a set of current shims to correct for the measured magnetic field residual gradients [43]. We are also developing a device consisting of a gas-filled quadrupole ion guide [55] in order to reduce the incident beam emittance and increase the sensitivity of the 
spectrometer. These improvements will allow MISTRAL to realize its full potential for accurate mass measurements of the shortest-lived nuclides approaching the drip line.

\section{ACKNOWLEDGMENTS}

We would like to acknowledge the expert technical assistance of M. Dedieu, M. Jacotin, J.-F. Képinski, and G. Le Scornet from CSNSM, G. Lebée from CERN, and
M. Duma from IAP. We would also like to acknowledge two colleagues who have moved on since their important involvement in the initial development of the spectrometer: A. Coc and R. Fergeau. We would like to thank D. Guillemaud-Mueller, B. Jonson, G. Hansen, H.-J. Kluge, R.B. Moore, A.C. Mueller, G. Nyman, and H. Wollnik for their support of the initial proposal to the ISOLDE committee in 1993. D.L. would like to acknowledge financial support from CERN.
[1] G. Audi and A.H. Wapstra, Nucl. Phys. A595, 409 (1995).

[2] C. Thibault, R. Klapisch, C. Rigaud, A.M. Poskanzer, R. Prieels, L. Lessard, and W. Reisdorf, Phys. Rev. 12, 644 (1975)

[3] C. Détraz, M. Langevin, M.C. Goffri-Kouassi, D. Guillemaud, M. Epherre, G. Audi, C. Thibault, and F. Touchard, Nucl. Phys. A394, 378 (1983).

[4] D.J. Vieira, J.M. Wouters, K. Vaziri, R.H. Krauss, Jr., H. Wollnik, G.W. Butler, F.K. Wohn, and A.H. Wapstra, Phys. Rev. Lett. 57, 3253 (1986).

[5] A. Gillibert, W. Mittig, L. Bianchi, A. Cunsolo, B. Fernandez, A. Foti, J. Gastebois, C. Grégoire, Y. Schutz, and C. Stéphan, Phys. Lett. B 192, 39 (1987).

[6] N.A. Orr, W. Mittig, L.K. Fifield, M. Lewitowicz, E. Plagnol, Y. Schutz, W.L. Zhan, L. Bianchi, A. Gillibert, A.V. Belozyorov, S.M. Lukyanov, Yu.E. Penionzhkevich, A.C.C. Villari, A. Cunsolo, A. Foti, G. Audi, C. Stéphan, and L. Tassan-Got, Phys. Lett. B 258, 29 (1991); 271, 468(E) (1991).

[7] X.G. Zhou, X.L. Tu, J.M. Wouters, D.J. Vieira, K.E.G. Lobner, H.L. Seifert, Z.Y. Zhou, and G.W. Butler, Phys. Lett. B 260, 285 (1991).

[8] G. Huber et al., Phys. Rev. C 18, 2342 (1978).

[9] C. Détraz, D. Guillemaud, G. Huber, R. Klapisch, M. Langevin, F. Naulin, C. Thibault, L.C. Carraz, and F. Touchard, Phys. Rev. C 19, 164 (1979).

[10] D. Guillemaud-Mueller, C. Détraz, M. Langevin, F. Naulin, M. de Saint Simon, C. Thibault, F. Touchard, and M. Epherre, Nucl. Phys. A426, 37 (1984).

[11] G. Klotz, P. Baumann, M. Bounajma, A. Huck, A. Knipper, G. Walter, G. Marguier, C. Richard-Serre, A. Poves, and J. Retamosa, Phys. Rev. C 47, 2502 (1993).

[12] T. Motobayashi, Y. Ikeda, Y. Ando, K. Ieki, M. Inoue, N. Iwasa, T. Kikuchi, M. Kurokawa, S. Moriya, S. Ogawa, H. Murakami, S. Shimoura, Y. Yanagisawa, T. Nakamura, Y. Watanabe, M. Ishihara, T. Teranishi, H. Okuno, and R.F. Casten, Phys. Lett. B 346, 9 (1995).

[13] S. Wilbert et al., in Proceedings of the International Conference on Exotic Nuclei and Atomic Masses (ENAM98), edited by B.M. Sherrill, D.J. Morrissey, and C.N. Davids, AIP Conf. Proc. No. 455, (AIP, New York, 1998), p. 142.

[14] M. Keim, in Proceedings of the International Conference on Exotic Nuclei and Atomic Masses (ENAM98) [13], p. 50.

[15] X. Campi, H. Flocard, A.K. Kerman, and S. Koonin, Nucl. Phys. A251, 193 (1975).

[16] J. Terasaki, H. Flocard, P.-H. Heenen, and P. Bonche, Nucl. Phys. A621, 706 (1997).
[17] W. Chung and B.H. Wildenthal, Phys. Rev. C 22, 2260 (1980).

[18] A. Poves and J. Retamosa, Phys. Lett. B 184, 311 (1987).

[19] E.K. Warburton, J.A. Becker, and B.A. Brown, Phys. Rev. C 41, 1147 (1990).

[20] N. Fukunishi, T. Otsuka, and T. Sebe, Phys. Lett. B 296, 279 (1992).

[21] E. Caurier, F. Nowacki, A. Poves, and J. Retamosa, Phys. Rev. C 58, 2033 (1998).

[22] S.K. Patra and C.R. Praharaj, Phys. Lett. B 273, 13 (1991).

[23] Z. Ren, Z.Y. Zhu, Y.H. Cai, and G. Xu, Phys. Lett. B 380, 241 (1996).

[24] W. Mittig, A. Lépine-Szily, and N.A. Orr, Annu. Rev. Nucl. Sci. 47, 27 (1997).

[25] B.V. Pritychenko et al., Phys. Lett. B 461, 322 (1999).

[26] F. Sarazin et al., Phys. Rev. Lett. 84, 5062 (2000); Ph.D. thesis, University of Caen, 1999.

[27] G. Bollen, Nucl. Phys. A626, 297c (1997).

[28] Proceedings of the 2nd Euroconference on Atomic Physics at Accelerators: Mass Spectrometry, Cargèse, France, edited by D. Lunney, G. Audi, and H.-J. Kluge [Hyperfine Interact. (to be published)].

[29] B. Fogelberg, K.A. Mezilev, H. Mach, V.I. Isakov, and J. Slivova, Phys. Rev. Lett. 82, 1823 (1999).

[30] X. Lu, J. Guo, K. Zhao, Y. Cheng, Y. Ma, Z. Li, S. Li, and M. Ruan, Eur. Phys. J. A 2, 149 (1998).

[31] G. Bollen et al., Nucl. Instrum. Methods Phys. Res. A 368, 675 (1996).

[32] B. Schlitt et al., Nucl. Phys. A626, 315c (1997).

[33] T. Radon et al., Phys. Rev. Lett. 78, 4701 (1997).

[34] H. Geissel et al., Nucl. Phys. A685, 115c (2001).

[35] C. Toader, Ph.D. thesis, Université Paris-Sud, Orsay, 1999; http://csnwww.in2p3.fr/AMDC/experimental/th-toader.pdf

[36] L.G. Smith, in Proceedings of the International Conference on Nuclidic Masses, edited by H. Duckworth (University of Toronto Press, Toronto, Canada, 1960), p. 418.

[37] L.G. Smith, in Proceedings of the 3rd International Conference on Atomic Masses, edited by R. Barber (University of Manitoba Press, Manitoba, Canada, 1967), p. 811.

[38] M. de Saint Simon, C. Thibault, G. Audi, A. Coc, H. Doubre, M. Jacotin, J.F. Képinski, R. Le Gac, G. Le Scornet, D. Lunney, and F. Touchard, Phys. Scr. T59, 406 (1995).

[39] M.D. Lunney, G. Audi, C. Borcea, M. Dedieu, H. Doubre, M. Duma, M. Jacotin, J.F. Képinski, G. Le Scornet, M. de Saint Simon, and C. Thibault, Hyperfine Interact. 99, 105 (1996).

[40] B. Jonson, H.L. Ravn, and G. Walter, Nucl. Phys. News 3, 5 (1993). 
[41] A. Coc, R. Le Gac, M. de Saint Simon, C. Thibault, and F. Touchard, Nucl. Instrum. Methods Phys. Res. A 271, 512 (1988).

[42] C. Monsanglant, Ph.D. thesis, Université Paris-Sud, Orsay, 2000; http://csnwww.in2p3.fr/AMDC/experimental/thmonsangl.pdf

[43] A. Coc, R. Fergeau, R. Grabit, M. Jacotin, J.F. Képinski, R. Le Gac, G. Le Scornet, G. Petrucci, M. de Saint Simon, G. Stefanini, C. Thibault, and F. Touchard, Nucl. Instrum. Methods Phys. Res. A 305, 143 (1991).

[44] G. Audi, A.H. Wapstra, and M. Dedieu, Nucl. Phys. A565, 193 (1993).

[45] E.R. Flynn and J.D. Garrett, Phys. Rev. C 9, 210 (1974).

[46] G.C. Ball, W.G. Davies, J.S. Forster, and J.C. Hardy, Phys. Rev. Lett. 28, 1069 (1972).

[47] D.E. Alburger, D.R. Goosman, and C.N. Davids, Phys. Rev. C
8, 1011 (1973).

[48] L.K. Fifield, C.L. Woods, R.A. Bark, P.V. Drumm, and M.A.C. Hotchkis, Nucl. Phys. A440, 531 (1985).

[49] I. Paschopoulos, E. Müller, H.J. Körner, I.C. Oelrich, K.E. Rehm, and H.J. Scheerer, Phys. Rev. C 18, 1277 (1978).

[50] S. Goriely, F. Tondeur, and J.M. Pearson, At. Data Nucl. Data Tables 77, 311 (2001).

[51] T. Otsuka and N. Fukunishi, Phys. Rep. 264, 297 (1996).

[52] Y. Utsono et al. (unpublished).

[53] Y. Aboussir, J.M. Pearson, A.K. Dutta, and F. Tondeur, At. Data Nucl. Data Tables 61, 127 (1995).

[54] P. Möller, J.R. Nix, W.D. Myers, and W.J. Swiatecki, At. Data Nucl. Data Tables 59, 185 (1995).

[55] M.D. Lunney and R.B. Moore, Int. J. Mass. Spectrom. 190/191, 153 (1999). 